\title{
Seleção do Par Dador-Recetor em Transplante Renal: Resultados Comparativos de uma Simulação
}

\author{
Selection of Donor-Recipient Pairs in Renal \\ Transplantation: Comparative Simulation Results
}

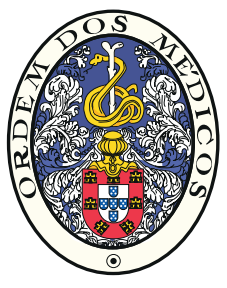

\author{
Bruno Alves LIMA $\triangle^{1}$, Helena ALVES ${ }^{2}$ \\ Acta Med Port 2017 Dec;30(12):854-860 - https://doi.org/10.20344/amp.8992
}

\section{RESUMO}

Introdução: Implementadas em 2007 pelo Despacho n 6357, as regras de alocação de rins de dador cadáver procuram distribuir de forma equitativa um bem escasso da comunidade destinado a doentes que com ele possam ver melhorada a sua sobrevivência e qualidade de vida. Tal como exposto no referido despacho estas regras devem ser atualizadas sempre que o estado da arte o recomendar. O objetivo deste trabalho é o de avaliar e comparar três modelos de alocação de rins de dador cadáver: critérios de pontuação das regras do despacho $n^{\circ} 6537 / 2007$ (modelo 1); modelo semelhante ao anterior mas com menor pontuação para o tempo de diálise (modelo 2); e um modelo adaptado do sistema de alocação por cores previamente proposto (modelo 3).

Material e Métodos: Para efeitos desta análise foram gerados dados para 70 dadores tendo em conta a informação publicada relativa à distribuição de grupo sanguíneo e de frequências alélicas e haplotípicas do sistema de antigénios leucocitários humanos de dadores voluntários portugueses. Foram também gerados dados para uma lista de espera simulada de 500 candidatos a primeiro transplante renal.

Resultados: Para a frequência do número de incompatibilidades de antigénios leucocitários humanos dos candidatos selecionados por cada modelo verifica-se que há menos candidatos no modelo $3 \mathrm{com}$ mais de 3 incompatibilidades de antigénios leucocitários humanos $(39,3 \%)$ do que no modelo $1(57,1 \%, p<0,01)$.

Discussão: Em comparação com as regras adaptadas do despacho n 6537/2007 (modelo 1) para alocação de rins, o modelo 3 seleciona candidatos com menor número de incompatibilidades de antigénios leucocitários humanos sem penalizar os candidatos com um maior tempo de diálise.

Conclusão: A análise e discussão das melhores regras a utilizar na alocação de um bem tão escasso como os órgãos de dador cadáver deve ser um processo contínuo e adaptável à evolução e mutação inerentes à lista de espera de candidatos a transplante.

Palavras-chave: Antígenos HLA; Obtenção de Tecidos e Órgãos; Portugal; Seleção do Doador; Teste de Histocompatibilidade; Transplantação Renal

\section{ABSTRACT}

Introduction: Implemented in 2007 by Ordinance No. 6357, allocation rules of cadaveric donor kidneys seek to distribute equitably a scarce community resource to patients who can improve their survival and quality of life. As stated in the aforementioned ordinance these rules must be updated whenever the state of the art recommends it. The objective of this work is to evaluate and compare three cadaveric donor allocation models: scoring criteria of ordinance $n^{\circ} 6537 / 2007$ (model 1); similar to the previous model but with a lower score for the dialysis time (model 2); and a model adapted from the previously proposed color allocation system (model 3 ).

Material and Methods: For the purpose of this analysis we generated data about 70 cadaveric donors taking into account information published regarding blood group distribution and human leucocyte antigens allelic and haplotype frequencies of Portuguese voluntary donors. We generated also data for a simulated waiting list of 500 first-time kidney transplant candidates.

Results: We observed fewer candidates selected by model 3 with more than 3 human leucocyte antigens mismatches (39.3\%) when compared to those selected by model 1 with more than 3 human leucocyte antigens mismatches $(57.1 \%, p<0.01)$.

Discussion: In our analysis, model 3 selects transplant candidates with a lower number of human leucocyte antigens mismatches when compared to the adapted rules for kidney allocation of Ordinance No. 6537/2007 (model 1) without penalizing candidates with a longer time on dialysis.

Conclusion: The analysis and discussion of the best rules for allocation of such a scarce resource as organs from deceased donors should be a continuous and adaptive process inherent to transplant candidate's waiting list evolution and mutation.

Palavras-chave: Donor Selection; Histocompatibility Testing; HLA Antigens; Kidney Transplantation; Portugal; Tissue and Organ Procurement

\section{INTRODUÇÃO}

Com o aumento da incidência de diabetes e hipertensão arterial e dado o envelhecimento da população, a doença renal crónica é uma doença cada vez mais frequente em todo o mundo. ${ }^{1}$ Devido à natureza assintomática da doença esta é frequentemente detetada em fases avançadas podendo progredir para doença renal crónica terminal em que o doente tem de ser submetido a terapêuticas de substituição renal como são a diálise ou o transplante. ${ }^{2}$

Quando comparado com a diálise, o transplante renal está associado a uma redução do risco de mortalidade bem como à redução de eventos cardiovasculares, sendo a melhor opção como tratamento de substituição da função renal sempre que possível. ${ }^{3}$ Os rins de dador cadáver para transplante são um bem escasso e consequentemente a

1. Oficina de Bioestatística. Ermesinde. Portugal.

2. Instituto Nacional de Saúde Dr. Ricardo Jorge. Porto. Portugal.

$\triangle$ Autor correspondente: Bruno Alves Lima. balima78@gmail.com

Recebido: 23 de março de 2017 - Aceite: 18 de outubro de 2017| Copyright @ Ordem dos Médicos 2017 
sua distribuição deve ser realizada da forma mais equitativa e transparente possível. ${ }^{4}$ A equidade na distribuição dos órgãos reside no equilíbrio entre uma distribuição justa, como seja, dar prioridade a quem espera há mais tempo por um órgão, e uma distribuição útil ou eficiente que passa por garantir que quem receba um órgão é quem tem as melhores condições para o receber [por exemplo, maior número de compatibilidades ao nível do sistema de antigénios leucocitários humanos (HLA)]. 5,6

Em Portugal, as regras de alocação de rim de dador cadáver foram implementadas em 2007 pelo despacho $\mathrm{n}^{\circ}$ 6357 e aplicam-se aos doentes candidatos a transplante renal em lista de espera (LE). Estes doentes são estudados periodicamente enquanto em lista de espera para que Ihes seja definido um perfil imunológico. Este obtém-se da sua genotipagem HLA e da análise periódica dos seus soros com a identificação de eventuais anticorpos anti-HLA. A identificação destes anticorpos anti-HLA não aceitáveis é feita através de técnicas de single antigen beads para valores de mean fluorescence intensity $(\mathrm{MFI}) \geq 1000 .^{7,8} \mathrm{Com}$ este perfil imunológico é possível efetuar um crossmatch virtual a cada candidato sempre que há um dador para transplante. Assim, a alocação dos rins é feita excluindo os candidatos com anticorpos específicos do dador (AED) (crossmatch virtual positivo) e selecionando aqueles que têm o mesmo grupo ABO do dador (distribuição iso-grupal). Posteriormente, aos candidatos são atribuídos pontos tendo em conta as incompatibilidades (mismatchs) HLA, o tempo em diálise, a idade, e o valor de painel reativo de anticorpos (PRA) por citotoxicidade dependente do complemento (CDC). ${ }^{7}$ Este sistema de alocação dos rins não tem em conta o valor calculado do painel reativo de anticorpos (cPRA). Este valor estima, para cada candidato a transplante, a probabilidade de um crossmatch virtual positivo tendo em conta as tipagens HLA de um determinado grupo de possíveis dadores. ${ }^{9} \mathrm{O}$ actual sistema de alocação de órgãos, para além de ser pouco transparente ${ }^{10}$ - uma vez que os doentes não têm como saber qual a sua posição relativa na LE - dá maior prioridade ao tempo em diálise em detrimento das compatibilidades HLA entre dador e recetor. ${ }^{11}$ As regras do despacho $n^{\circ} 6537 / 2007$ foram também associadas com um maior número de episódios de rejeição, ${ }^{12}$ no entanto, não temos conhecimento de que estes resultados tenham sido replicados em publicações posteriores.

Como alternativa às atuais regras de distribuição de rins de dador cadáver, sugerimos em 2013, um sistema de classificação por cores. ${ }^{10}$ Este sistema prioriza os candidatos a transplante em LE tendo em conta o seu tempo em diálise e a sua sensibilização a anticorpos anti HLA.

O objetivo deste trabalho é o de avaliar e comparar três modelos de alocação de rins de dador cadáver. O primeiro modelo é adaptado directamente dos critérios de pontuação das regras do despacho n 6537/2007, o segundo modelo difere do primeiro apenas na pontuação dada ao tempo em diálise e o terceiro modelo é adaptado do sistema de alocação por cores previamente proposto. ${ }^{10}$

\section{MATERIAL E MÉTODOS}

Para efeitos desta análise foram gerados dados para 70 dadores cujas idades foram atribuídas por valores aleatórios duma distribuição normal de média 55 anos e desvio padrão 15, truncados entre os valores 18 e 75 . Os parâmetros desta distribuição normal foram escolhidos arbitrariamente e traduzem a experiência dos autores. As frequências para os respectivos grupos sanguíneos foram atribuídas aleatoriamente a cada um dos dadores tendo em conta as frequências previamente descritas para dadores de sangue em Portugal. ${ }^{13}$ Assim, 32 dadores são do grupo A, 6 do grupo B, 3 do grupo AB e 29 do grupo O. Os dados relativos à tipagem $\mathrm{HLA}$ de cada um destes dadores foram gerados tendo em conta as frequências alélicas e haplotípicas descritas para dadores voluntários de medula óssea do Norte de Portugal. ${ }^{14}$

Foram também gerados dados para uma lista de espera simulada de 500 doentes candidatos a primeiro transplante renal. As idades destes doentes são 500 valores aleatórios duma distribuição normal de média 45 anos e desvio padrão 15, truncados entre os valores 18 e 75 . As frequências para o grupo $\mathrm{ABO}$ foram definidas para que o grupo $\mathrm{O}$ com 46\% (230) seja o mais frequente; do grupo A são $43 \%$ (215), do grupo B são 8\% (40) e do grupo AB são 3\% (15) dos candidatos. Os dados relativos à tipagem HLA de cada um destes candidatos foram gerados tendo em conta as frequências alélicas e haplotipicas descritas para dadores voluntários de medula óssea do Norte de Portugal. ${ }^{14}$

Foi atribuído aleatoriamente a cada candidato um grupo de cPRA segundo as frequências: $80 \%$ dos doentes com valores $0 \%$ de cPRA; $5 \%$ dos doentes com valores no intervalo $(10 \% ; 40 \%) ; 5 \%$ com valores no intervalo $(40 \% ; 80 \%)$; e $10 \%$ com valores $\geq 80 \%$. Para os doentes dos grupos de cPRA maior que $0 \%$ foram atribuídos anticorpos anti-HLA (obrigatoriamente diferentes dos respectivos antigénios HLA) de modo a calcular a percentagem dos 70 dadores simulados que tenham antigénios HLA coincidentes com esses anticorpos. O cPRA de cada um destes candidatos a transplante é assim igual à percentagem de dadores com

Tabela 1 - Sistema de alocação por cores (modelo 3)

\begin{tabular}{|c|c|c|c|c|}
\hline & Dador $\leq 65$ anos & & Dador $>65$ anos & \\
\hline \multirow{4}{*}{ 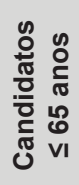 } & Urgência clínica & Vermelho & Urgência clínica & \multirow{4}{*}{ 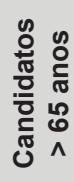 } \\
\hline & cPRA $\geq 85 \%$ ou TD $\geq 3^{\circ}$ Quartil & Laranja & cPRA $\geq 85 \%$ ou TD $\geq 3^{\circ}$ Quartil & \\
\hline & cPRA $\geq 50 \%$ ou TD $\geq$ Mediana & Amarelo & cPRA $\geq 50 \%$ ou TD $\geq$ Mediana & \\
\hline & cPRA $<50 \%$ e TD $<$ Mediana & Verde & cPRA $<50 \%$ e TD $<$ Mediana & \\
\hline
\end{tabular}

cPRA: valor calculado de pesquisa de reatividade alogénica; TD: tempo de diálise 
os quais terão um crossmatch virtual positivo.

O tempo de diálise de cada um dos candidatos foi gerado aleatoriamente tendo em conta o grupo $A B O$ e o valor de cPRA atribuído a cada um deles. Assim para os doentes do grupo $\mathrm{O}$ e com valores de cPRA $\geq 80 \%$ foram gerados valores com distribuição normal de média 70 meses e desvio padrão 20, para doentes que apenas tenham uma destas características (grupo O ou cPRA $\geq 80 \%$ ) foram gerados valores com distribuição normal com média de 55 meses e desvio padrão 20 , para os restantes doentes foram gerados valores aleatórios com distribuição normal com média 40 meses e desvio padrão 20.

A cada um dos doentes candidatos a transplante foi também atribuída uma cor de acordo com o descrito na Tabela 1 para o sistema de cores de alocação de rins de dadores cadáver.

As frequências e distribuições dos valores gerados para os 500 candidatos simulados foram definidas arbitrariamente e traduzem a experiência dos autores, tendo em conta a informação disponível para a lista de espera de candidatos a transplante renal em Portugal. ${ }^{15}$

Para cada um dos modelos em análise, os dadores (um a um) foram alocados aos dois melhores recetores possíveis (aplicando as regras de cada modelo) de forma a obterem-se grupos de até 140 recetores por modelo e por simulação. Assim, para o modelo 1 e para o primeiro dador, dos 500 candidatos foram selecionados aqueles que tinham o mesmo grupo sanguíneo do dador e que com este tinham um crossmatch virtual negativo, aos selecionados foi calculada a respectiva pontuação (conforme descrito na Tabela 2) e aos dois doentes com a pontuação mais elevada foi-lhes alocado o primeiro dador; para o segundo dador seguiu-se o mesmo procedimento para os 498 candidatos disponíveis e assim sucessivamente até serem alocados os 70 dadores a um máximo de 140 recetores.

Tabela 2 - Tabela de pontuação do despacho n 6357/2007, modificada para efeitos de análise (modelo 1)

\begin{tabular}{lc}
\hline Critério & Pontos \\
\hline Incompatibilidades HLA* & 12 \\
a) Sem incompatibilidades em A, B e DR & 8 \\
b) Sem incompatibilidades em B e DR & 4 \\
c) Uma incompatibilidade em B ou DR & 2 \\
d) Uma incompatibilidade em B e uma em DR & 1 \\
e) Restantes possibilidades & 8 \\
cPRA $\geq 85 \%$ & 4 \\
cPRA $\geq 50 \%$ & 0,1 \\
Tempo de diálise (cada mês) & \\
Diferença de idade entre dador e recetor & 0 \\
Dador $>60$ anos e recetor < 55 anos & 0 \\
Dador < 40 anos e recetor $>55$ anos & 4 \\
Restantes possibilidades &
\end{tabular}

*As incompatibilidades para o locus HLA-A servem como critério de desempate em caso de igualdade pontual; HLA: antigénios leucocitários humanos; CPRA: valor calculado para a pesquisa de reatividade alogénica
Para o modelo 2 o procedimento foi semelhante aplicando-se apenas 0,05 pontos por cada mês de diálise, em vez de 0,1 pontos atribuídos no modelo 1 .

O modelo por cores, previamente proposto, procura um equilíbrio entre uma distribuição justa e eficiente de bens escassos, como são os rins de dadores cadáver. ${ }^{10}$ Uma distribuição justa dos rins seria baseada principalmente no tempo de diálise dos candidatos a transplante, enquanto uma distribuição eficiente ou útil daria o primado ao número de compatibilidades HLA entre dador e candidato. O modelo 3 , aqui analisado, começa por classificar os doentes em LE por cores tendo em conta a sua urgência clínica (no caso do vermelho) ou o seu tempo de diálise e valor de cPRA para as restantes cores do sistema (laranja, amarelo e verde) (Tabela 1). Como laranja são classificados os doentes em LE com valores de cPRA $\geq 85 \%$ ou com um tempo de diálise maior que o terceiro quartil do tempo de diálise até ao transplante (isto é, o tempo em diálise necessário até $75 \%$ dos candidatos serem transplantados). Como amarelos são classificados os candidatos com cPRA $\geq 50 \%$ ou com um tempo de diálise maior que a mediana do tempo de diálise para transplante (isto é, o tempo de diálise necessário para $50 \%$ dos doentes em LE serem transplantados). Como verdes são classificados os restantes doentes na lista de espera. Neste modelo de cores, para cada dador disponível para transplante foram selecionados inicialmente os candidatos isogrupais com crossmatch virtual negativo e que pertenciam à mesma classe etária, isto é, caso o dador tivesse mais de 65 anos apenas foram selecionados os candidatos com mais de 65 anos enquanto para dadores menores que 65 apenas foram selecionados os candidatos menores de 65 anos. Posteriormente foram calculadas as

Tabela 3 - Dados demográficos e imunológicos dos 500 candidatos em lista de espera

\begin{tabular}{lcc}
\hline & $\begin{array}{c}n \\
(\%)\end{array}$ & $\begin{array}{c}\text { Mediana } \\
\left(1^{\circ} \mathrm{Q}-3^{\circ} \mathrm{Q}\right)\end{array}$ \\
\hline Grupo ABO & \\
A & $215(43)$ & \\
AB & $15(3)$ & \\
B & $40(8)$ & \\
O & $230(46)$ & $46(36-55)$ \\
Idade (anos) & \\
Tempo de diálise (meses) & \\
cPRA & \\
$0 \%$ & $400(81-62)$ \\
$(0 \% ; 50 \%)$ & $34(6,8)$ \\
$(50 ; 85 \%)$ & $43(8,6)$ \\
$\geq 85 \%$ & $23(4,6)$ \\
Cor * & \\
Laranja & $143(28,6)$ \\
Amarelo & $134(26,8)$ \\
Verde & $223(44,6)$ \\
\hline
\end{tabular}

Q: quartil; cPRA: valor calculado para a pesquisa de reatividade alogénica * classificação por cores de acordo com o sistema de alocação proposto 
incompatibilidades (mismatches) HLA (mmHLA) de cada doente e estes foram ordenados primeiro por prioridade de cor e posteriormente por número crescente de incompatibilidades. Em caso de empates, foram selecionados os doentes com maior tempo de diálise. Aos dois candidatos com menor número de incompatibilidades no grupo de cor mais prioritário foi-Ihes alocado o correspondente dador.

A comparação das medianas do tempo de diálise e idade dos recetores selecionados para cada modelo foi feita através do teste de Kruskal-Wallis. O teste de qui-quadrado (ou teste exacto de Fisher, quando apropriado) foi usado para comparar as frequências de recetores por grupo de cPRA e grupos de mismatches HLA entre os modelos. Os valores de $p<0,05$ foram considerados estatisticamente significativos.

Todas as análises estatísticas tal como as representações gráficas foram realizadas com recurso ao software RStudio para a linguagem e ambiente de programação R.

\section{RESULTADOS}

As características demográficas e clínicas da lista de espera composta pelos 500 doentes candidatos a transplante simulados estão descritas na Tabela 3. Quase metade dos candidatos $(44,6 \%)$ em lista de espera foram classificados como verdes, enquanto $28,6 \%$ foram classificados como laranja.

As idades dos candidatos selecionados em cada um dos modelos são muito semelhantes apresentando medianas de 46 anos no modelo 1 e de 47 anos para os outros dois modelos (Tabela 4).

Comparadas as medianas dos tempos de diálise dos recetores selecionados através de cada um dos modelos, verificamos que não existem diferenças estatisticamente significativas entre o modelo 2 (mediana de 62,5 meses) e o modelo 1 (mediana de 67 meses), nem entre o modelo 3 (mediana de 67 meses) e o modelo 1, embora os recetores do modelo 2 sejam os que apresentam valores de tempo de diálise mais baixos e de mais amplitude. Para a frequência do número de mismatches HLA dos recetores selecionados por modelo verifica-se que o modelo 3 selecciona menos recetores com vários mismatches HLA. Verificamos que há menos doentes no modelo $3 \mathrm{com}$

Tabela 4 - Comparação das características dos candidatos selecionados através de cada um dos modelos de alocação em análise

\begin{tabular}{|c|c|c|c|c|c|c|c|c|}
\hline & \multicolumn{2}{|c|}{ Modelo 1} & \multicolumn{2}{|c|}{ Modelo 2} & \multirow[b]{2}{*}{$p^{*}$} & \multicolumn{2}{|c|}{ Modelo3 } & \multirow[b]{2}{*}{$p^{\dagger}$} \\
\hline & $\mathrm{n}(\%)$ & $\begin{array}{c}\text { Mediana } \\
\left(1^{\circ} \mathrm{Q}-3^{\circ} \mathrm{Q}\right)\end{array}$ & $\mathrm{n}(\%)$ & $\begin{array}{c}\text { Mediana } \\
\left(1^{\circ} \mathrm{Q}-3^{\circ} \mathrm{Q}\right)\end{array}$ & & n (\%) & $\begin{array}{c}\text { Mediana } \\
\left(1^{\circ} \mathrm{Q}-3^{\circ} \mathrm{Q}\right)\end{array}$ & \\
\hline Idade (anos) & & $\begin{array}{c}46,0 \\
(36,8-55,0)\end{array}$ & & $\begin{array}{c}47,0 \\
(37,0-55,0)\end{array}$ & 0,857 & & $\begin{array}{c}47,0 \\
(37,0-56,5)\end{array}$ & 0,529 \\
\hline TD (meses) & & $\begin{array}{c}67,0 \\
(51,5-81,0)\end{array}$ & & $\begin{array}{c}62,5 \\
(46,8-79,0)\end{array}$ & 0,103 & & $\begin{array}{c}67,0 \\
(58,0-80,5)\end{array}$ & 0,43 \\
\hline mmHLA & & & & & 0,525 & & & 0,009 \\
\hline 1 & $8(5,7)$ & & $10(7,1)$ & & & $4(2,9)$ & & \\
\hline 2 & $21(15,0)$ & & $24(17,1)$ & & & $22(15,8)$ & & \\
\hline 3 & $31(22,1)$ & & $41(29,3)$ & & & $58(41,7)$ & & \\
\hline 4 & $42(30,0)$ & & $30(21,4)$ & & & $32(23,0)$ & & \\
\hline 5 & $24(17,1)$ & & $24(17,1)$ & & & $17(12,2)$ & & \\
\hline 6 & $14(10,0)$ & & $11(7,9)$ & & & $6(4,3)$ & & \\
\hline Até $3 \mathrm{mmHLA}$ & $60(42,9)$ & & $75(53,6)$ & & 0,073 & $84(60,4)$ & & 0,003 \\
\hline Mais de $3 \mathrm{mmHLA}$ & $80(57,1)$ & & $65(46,4)$ & & & $55(39,6)$ & & \\
\hline $\mathrm{mmBDR}$ & & & & & 0,573 & & & $<0,01$ \\
\hline 0 & $7(5,0)$ & & $9(6,4)$ & & & $0(0)$ & & \\
\hline 1 & $34(24,3)$ & & $41(29,3)$ & & & $20(14,4)$ & & \\
\hline 2 & $35(25,0)$ & & $38(27,1)$ & & & $68(48,9)$ & & \\
\hline 3 & $38(27,1)$ & & $27(19,3)$ & & & $36(25,9)$ & & \\
\hline 4 & $26(18,6)$ & & $25(17,9)$ & & & $15(10,8)$ & & \\
\hline Até 2 mmBDR & $76(54,3)$ & & $88(62,9)$ & & 0,145 & $88(63,3)$ & & 0,126 \\
\hline Mais de 2 mmBDR & $64(45,7)$ & & $52(37,1)$ & & & $51(36,7)$ & & \\
\hline cPRA & & & & & 0,685 & & & 0,001 \\
\hline$(0 ; 50)$ & $82(58,6)$ & & $77(55,0)$ & & & $112(80,6)$ & & \\
\hline$(50 ; 85)$ & $37(26,4)$ & & $42(30,0)$ & & & $14(10,1)$ & & \\
\hline$\geq 85$ & $21(15,0)$ & & $21(15,0)$ & & & $13(9,4)$ & & \\
\hline
\end{tabular}

* valor de $p$ para a comparação entre o modelo 1 e o modelo 2; † valor de $p$ para a comparação entre o modelo 1 e o modelo 3; Q: Quartil; TD: Tempo de diálise; HLA: Antigénios leucocitários humanos; mmHLA: número de mismatches em HLA-A*, - $B^{*}$ e -DRB1*; mmBDR: número de mismatches em HLA-B* e -DRB1*; cPRA: valor calculado de pesquisa de reatividade alogénica 
mais de 3 mmHLA $(39,3 \%)$ do que no modelo $1(57,1 \%)$ sendo esta diferença estatisticamente significativa $(p<$ $0,01)$. Também no modelo 2 são menos os doentes com mais de 3 mmHLA $(46,4 \%)$ do que no modelo 1 , no entanto, neste caso a diferença não é estatisticamente significativa. Quando são consideradas apenas as incompatibilidades apenas nos loci $\mathrm{B}^{*}$ e DRB1* verificamos que no modelo 1 são mais os doentes com mais de 2 incompatibilidades em B e DRB1 $(45,7 \%)$ do que aqueles selecionados nos modelos 2 e $3(37,1 \%$ e $34,4 \%$, respectivamente), mas estas diferenças não são estatisticamente significativas. Para a distribuição dos recetores selecionados em cada modelo pelos grupos de cPRA, verificamos que o modelo 3 é o que seleciona um menor número de candidatos com valores de cPRA $\geq 85 \%(9,4 \%)$ quando comparado com os outros dois modelos (15\%); sendo a diferença com o modelo 1 estatisticamente significativa $(p<0,01)$.

\section{DISCUSSÃO}

O modelo de classificação por cores (modelo 3) prioriza de uma forma mais transparente todos os candidatos em lista de espera para transplante de rim, tendo em conta o respectivo tempo de espera de cada candidato bem como a sua probabilidade de não ser selecionado para um futuro dador (através do cPRA).

Em comparação com as regras adaptadas do despacho $n^{\circ} 6537 / 2007$ (modelo 1) para alocação de rins, o modelo 3 seleciona candidatos com menor número de incompatibilidades HLA sem penalizar os candidatos com um maior tempo de diálise. No modelo 2 foi atribuído um menor peso ao tempo de diálise $(0,05$ pontos por mês de dialise $)$ do que para o modelo 1 ( 0,1 pontos por mês) e como consequência directa são selecionados candidatos com menor tempo de diálise, no entanto, não há um ganho estatisticamente significativo em termos da redução de incompatibilidades HLA entre dador e recetor.

As regras de alocação de rins de dador cadáver devem selecionar candidatos a transplante de modo a procurar um equilíbrio entre o tempo de espera dos candidatos (e/ ou o tempo em diálise) e garantias de sucesso imunológico do transplante (que se podem caucionar, tanto quanto possível, minimizando o número de transplantes com um elevado número de mismatchs HLA). Com a diminuição do número de transplantes realizados em Portugal a partir do ano de $2012^{16}$ torna-se ainda mais premente definir regras que possam viabilizar um maior sucesso do transplante. Nesta análise, o modelo 3 proposto para alocação de rins de dador cadáver é o que melhor traduz este desejado equilíbrio.

O modelo 3, pelo facto de usar cores para classificar prioritariamente os candidatos em LE para transplante é um modelo mais simples e inteligível (também para os próprios doentes) do que um modelo por pontos (visto como uma caixa negra difícil de decifrar). Ao atribuirmos uma cor que traduz um grau de prioridade a cada um dos doentes em LE, não estamos a afirmar que o doente $A$, classificado com a cor laranja, será transplantado primeiro que o doente
B, classificado com a cor verde; mas podemos afirmar que os doentes laranjas, em média, demorarão menos tempo a ser transplantados do que os doentes verdes. No caso da nossa simulação, verificamos que dos candidatos selecionados pelo modelo 3, 69,8\% estavam classificados com a cor laranja, enquanto que apenas $1,4 \%$ estavam classificados como verdes. Assim, podemos também afirmar que o modelo 3 é mais transparente do que o modelo actualmente em vigor.

$\mathrm{Na}$ seleção de candidatos para um possível dador impôs-se à priori (à semelhança das regras do despacho $n^{\circ}$ 6537/2007) a coincidência iso-grupo $A B O$ e que os candidatos não tenham anticorpos anti-HLA anti-dador (crossmatch virtual) de modo a minimizar riscos conhecidos de rejeição do órgão. A definição do perfil imunológico de cada candidato em LE para transplante é essencial para que se possam definir os resultados de crossmatch virtual com os possíveis dadores. O valor de $1000 \mathrm{MFI}$ para identificação desses anticorpos tem sido descrito como de elevada sensibilidade, o que se traduz na possibilidade de identificar anticorpos anti-HLA com um risco clínico de rejeição reduzido. Embora tenha sido sugerido que este cut-off de MFI deva ser aumentado para a identificação dos anticorpos de risco, dever-se-iam considerar os valores cumulativos de $\mathrm{MFI}$ (e não só os valores para cada anticorpo) para concluir sobre um crossmatch virtual positivo. ${ }^{17,18}$ Isto é, para além de considerar um crossmatch virtual positivo sempre que um candidato tenha um anticorpo anti-HLA específico do possível dador > $3000 \mathrm{MFI}$, também devem ser considerados crossmatch virtual positivos se o candidato apenas tem 3 AED com valores de $1000 \mathrm{MFI}$ cada um. Este mesmo princípio deverá ser aplicável ao valor cPRA, ou seja, neste cálculo deverão ser considerados os valores cumulativos de AED superiores a 3000 MFIs. O crossmatch virtual e os valores de cPRA devem ser vistos como duas faces da mesma moeda e são ferramentas essenciais para a seleção dos melhores candidatos a transplante para um determinado dador. ${ }^{19}$ As frequências genotípicas usadas para o cPRA devem corresponder, tanto quanto possível, com aquelas dos que serão os futuros dadores de órgãos e como tal devem ser conhecidas e escrutináveis ${ }^{14}$ e não pertencerem a um qualquer grupo escolhido ad hoc.

O despacho $n^{\circ} 6537 / 2007$ atribui pontos extra para valores de PRA por CDC maiores que 50\%, no entanto estes valores de PRA por CDC tendem a subestimar o número de crossmatchs positivos com futuros dadores. ${ }^{19,20} \mathrm{Na}$ análise aqui efetuada foram atribuídos anticorpos anti-HLA aos candidatos e posteriormente definidos os respectivos valores de cPRA e verificou-se que com o modelo 1 são selecionados mais candidatos com valores de cPRA $\geq$ $85 \%$ do que com o modelo 3 . Os doentes hipersensibilizados em LE são um desafio para qualquer programa de alocação de rins. Estes doentes tendem a esperar mais tempo por um órgão compatível aumentando assim o seu risco de comorbilidade e/ou de mortalidade, diminuindo a sua probabilidade de transplante. ${ }^{21}$ Já passaram mais de 25 anos da implementação pelo Eurotransplant do seu 
programa de mismatches aceitáveis para aumentar o número de transplantes de doentes hipersensibilizados. ${ }^{22} \mathrm{Em}-$ bora esta medida não seja uma panaceia que resolve todos os problemas, também em Portugal deve ser discutida a possibilidade de implementar um programa deste tipo para dar resposta a este problema. No modelo 3, os candidatos com um valor de cPRA $\geq 85 \%$ são classificados com a cor laranja, juntamente com os candidatos que, não sendo hipersensibilizados, têm os valores mais altos de tempo de diálise. No momento de ordenar estes candidatos laranja pelo número crescente de mismatchs com um possível dador, os doentes hipersensibilizados (a maioria com tipagem HLA menos comum uma vez que, por definição de cPRA tendem a ter anticorpos a HLA mais comuns) que não foram excluídos por crossmatch positivo, tendem a ser os que têm maior número de mismatchs e consequentemente são preteridos mais uma vez para transplante. Na procura de um equilíbrio entre uma distribuição justa e uma distribuição útil de rins, que está na génese do modelo 3 , os doentes hipersensibilizados são prejudicados e eventualmente tenderão a acumular-se em LE, uma vez que só são selecionados quando têm um número reduzido de mismatchs. Face ao exposto, deveremos no futuro considerar a possibilidade de acrescentar um primeiro nível de prioridade na alocação dos órgãos para os candidatos hipersensibilizados, entre as cores vermelha e laranja.

O benefício na sobrevivência dos doentes com transplante renal bem-sucedido, em comparação com aqueles que permanecem em diálise, é independente da idade dos seus dadores cadáveres. ${ }^{23}$ Deve referir-se também que a idade do dador é um factor associado com um pior prognóstico na sobrevivência do enxerto e do doente transplantado. ${ }^{24}$ As mudanças inerentes ao envelhecimento, como a diminuição do volume de filtração glomerular ou o aumento da imunogenicidade de órgãos mais velhos, podem ter impacto nos outcomes dos transplantes. ${ }^{25}$

A utilização de dadores de critérios expandidos pode permitir o aumento do número total de dadores disponíveis para transplante e consequente diminuição dos tempos de espera para transplante, mas deve ser gerida com cuidado. O transplante renal de doentes de idades mais avançadas com dadores cadáver mais idosos é uma boa alternativa para os primeiros. O benefício em termos de sobrevivência deste tipo de transplante em doentes mais velhos quando comparados com os doentes que permanecem em diálise existe e pode ser aproveitado. ${ }^{26,27}$ Tendo isto em conta, o modelo de cores aqui proposto impõe que dadores de maior idade sejam dirigidos a candidatos mais idosos, no entanto não encontramos diferenças estatisticamente significativas entre as idades dos candidatos selecionados com o modelo 1 e os candidatos selecionados com o modelo 3.

Com o aumento do número de candidatos a retransplante após rejeição do primeiro enxerto, ${ }^{28}$ novas regras de alocação de órgãos deverão também ter este grupo de doentes em consideração. O benefício de um retransplante em candidatos de baixo risco é semelhante ao benefício de um primeiro transplante, sendo o tempo de espera para o retransplante um dos principais factores de risco para o insucesso do transplante. ${ }^{29} \mathrm{Na}$ análise aqui efectuada apenas consideramos candidatos a primeiro transplante, por conveniência da nossa simulação.

Os resultados aqui apresentados foram obtidos através de dados simulados para uma hipotética LE de candidatos a transplante e um grupo de possíveis dadores cadáver Pese embora algumas iniciativas recentes de que é exemplo a criação do Portal do Serviço Nacional de Saúde, ${ }^{30}$ ainda não existem dados abertos ${ }^{31}$ referentes ao acesso ao transplante de rim que nos permitam testar com dados reais os modelos apresentados.

Alertados para os relatórios disponibilizados pela Sociedade Portuguesa de Nefrologia ${ }^{32}$ e pela Sociedade Portuguesa de Transplantação, ${ }^{33}$ constatamos que estas informações não são acompanhadas com dados abertos, que permitam uma avaliação analítica e cuidada das mesmas. Dados e informações nacionais relativos a: tempo de diálise até ao transplante; mediana do tempo de espera para o transplante; ou o número de dadores e transplantes por grupos etários ou unidade de transplante ${ }^{11,34}$ são apenas alguns exemplos de indicadores que não estão publicados em qualquer revista científica. A possibilidade de análise de dados usando técnicas estatísticas e metodológicas erradas, ou dando uma má interpretação de resultados, ou reportando resultados de forma selectiva são fenómenos há muito identificados, ${ }^{35}$ mas que agora podem ser minimizados com a disponibilização pública dos dados.

O número de candidatos em LE para transplante com dador cadáver resulta do fluxo de entradas (novas inscrições em LE e reinscrições de doentes com rejeição de transplantes prévios) e do fluxo de saídas da lista (transplantes realizados, saída de lista por razões clínicas, por vontade do próprio ou pela morte de candidatos enquanto em espera). Particularmente em Portugal, cada doente pode inscrever-se em duas unidades de transplante (ou seja, duas LE). Infelizmente, não está acessível informação mais pormenorizada, que nos permita analisar com maior detalhe a evolução da LE. Dados relativos a reinscrições em LE, após o primeiro transplante ou a percentagem de doentes que necessitam de repetir transplante e o peso destes doentes em LE também não estão disponíveis. A melhor forma de distribuir os rins de dador cadáver terá sempre de depender duma correcta caracterização da LE para transplante.

\section{CONCLUSÃO}

Em conclusão, um modelo de alocação de órgãos baseado em níveis de prioridade atribuídos aos candidatos a transplante e com a seleção destes com base nas incompatibilidades HLA pode ser um modelo mais simples, mais transparente e mais equitativo do que o modelo em vigor desde 2007. Tanto quanto é do nosso conhecimento, este é o primeiro trabalho que avalia objectivamente as atuais normas de selecção do par dador-recetor. A análise e discussão das melhores regras a utilizar na alocação de um 
bem tão escasso como os órgãos de dador cadáver deve ser um processo contínuo e adaptável à evolução e mutação inerentes à lista de espera de candidatos a transplante.

\section{PROTECÇÃO DE PESSOAS E ANIMAIS}

Os autores declaram que os procedimentos seguidos estavam de acordo com os regulamentos estabelecidos pelos responsáveis da Comissão de Investigação Clínica e Ética e de acordo com a Declaração de Helsínquia da Associação Médica Mundial.

\section{REFERÊNCIAS}

1. Vinhas J, Gardete-Correia L, Boavida JM, Raposo JF, Mesquita A, Fona $\mathrm{MC}$, et al. Prevalence of chronic kidney disease and associated risk factors, and risk of end-stage renal disease: data from the PREVADIAB study. Nephron Clin Pr. 2011;119:35-40.

2. Zhang Q, Rothenbacher D. Prevalence of chronic kidney disease in population-based studies: systematic review. BMC Public Health. 2008;8

3. Tonelli M, Wiebe N, Knoll G, Bello A, Browne S, Jadhav D, et al. Systematic review: Kidney transplantation compared with dialysis in clinically relevant outcomes. Am J Transplant. 2011;11:2093-109.

4. Pussell BA, Bendorf A, Kerridge $\mathrm{IH}$. Access to the kidney transplant waiting list: a time for reflection. Intern Med J. 2012;42:360-3.

5. Persijn GG. Allocation of organs, particularly kidneys, within Eurotransplant. Hum Immunol. 2006;67:419-23.

6. Freeman RB, Freeman RB. Survival benefit : quality versus quantity and trade-offs in developing new renal allocation systems. Am J Transpl. 2007;7:1043-6.

7. Tafulo S, Malheiro J, Dias L, Mendes C, Osório E, Martins LS, et al. Low transplantability of 0 blood group and highly sensitized candidates in the Portuguese kidney allocation algorithm : quantifying an old problem in search of new solutions. HLA. 2016;88:232-8.

8. Singh N, Djamali A, Lorentzen D, Pirsch JD, Leverson G, Neidlinger N et al. Pretransplant donor-specific antibodies detected by single-antigen bead flow cytometry are associated with inferior kidney transplant outcomes. Transplantation. 2010;90:1079-84.

9. Cecka JM. Calculated PRA (CPRA): the new measure of sensitization for transplant candidates: Special feature. Am J Transplant. 2010;10:269.

10. Lima BA, Mendes M, Alves H. Kidney transplant allocation in Portugal. Port J Nephrol Hypert. 2013;27:313-6.

11. Lima BA, Mendes $\mathrm{M}$, Alves $\mathrm{H}$. Kidney transplantation in the north of Portugal: donor type and recipient time on dialysis. Port $\mathrm{J}$ Nephrol Hypert. 2013;27:23-30.

12. Gonçalves JA, Jorge C, Atalaia A, Matias P, Bruges M, Birne R, et al. New law of renal transplantation in Portugal associated with more acute rejection episodes and higher costs. Transplant Proc. 2012;44:2276-9.

13. Duran J, Chabert T, Rodrigues F, Pestana D. Distribuição dos grupos sanguíneos na população portuguesa. AB0. 2007;29:5-17.

14. Lima BA, Alves H. HLA-A, -C, -B, AND -DRB1 alleleic and haplotypic diversity in bone marrow volunteer donors from northern Portugal. Organs Tissues Cells. 2013:19-26.

15. Lima BA. Acesso ao transplante de rim de dador cadáver no norte de Portugal. Dissertação de Mestrado em Saúde Pública. Faculdade de Medicina da Universidade do Porto. 2011. [consultado 2017 mar 9]. Disponível em: http://hdl.handle.net/10216/22118.

16. Lima BA, Alves $H$. Evolução da atividade de transplantação renal em Portugal: dados públicos de 2003 a 2015. Obs - Bol Epidemiol. 2017;18:24-7.

17. Lefaucheur C, Loupy A, Hill GS, Andrade J, Nochy D, Antoine C, et al. Preexisting donor-specific HLA antibodies predict outcome in kidney transplantation. J Am Soc Nephrol. 2010;21:1398-406.

18. Malheiro J, Tafulo S, Dias L, Martins LS, Fonseca I, Beirão I, et al. Analysis of preformed donor-speci fi c anti-HLA antibodies characteristics

\section{CONFIDENCIALIDADE DOS DADOS}

Os autores declaram ter seguido os protocolos do seu centro de trabalho acerca da publicação de dados.

\section{CONFLITOS DE INTERESSE}

Os autores declaram não terem qualquer conflito de interesse relativamente ao presente artigo.

\section{FONTES DE FINANCIAMENTO}

Os autores declaram não ter recebido subsídios ou bolsas para a elaboração do artigo.

for prediction of antibody-mediated rejection in kidney transplantation. Transpl Immunol. 2015;32:66-71.

19. Lima BA, Mendes M, Alves $H$. Hypersensitized candidates to kidney transplantation in Portugal. Port J Nephrol Hypert. 2013;27:77-81.

20. Magriço R, Malheiro J, Tafulo S, Pedroso S, Almeida M, Martins LS, et al. Implications for patients waiting for a kidney transplant of using the calculated panel reactive antibody (cPRA). Port J Nephrol Hypert. 2016;30:185-93.

21. Koefoed-Nielsen P, Weinreich I, Bengtsson M, Lauronen J, Naper C, Gäbel M, et al. Scandiatransplant acceptable mismatch program ( STAMP ) a bridge to transplanting highly immunized patients. HLA. 2017;90:17-24.

22. Heidt S, Witvliet MD, Haasnoot GW, Claas FHJ. The 25th anniversary of the Eurotransplant Acceptable Mismatch program for highly sensitized patients. Transpl Immunol. 2015;33:51-7.

23. Ojo AO, Hanson JA, Meier-Kriesche H, Okechukwu CN, Wolfe RA, Leichtman $A B$, et al. Survival in recipients of marginal cadaveric donor kidneys compared with other recipients and wait-listed transplant candidates. J Am Soc Nephrol. 2001;12:589-97.

24. Oppenheimer F, Aljama P, Asensio Peinado C, Bustamante Bustamante J, Crespo Albiach JF, Guirado Perich L. The impact of donor age on the results of renal transplantation. Nephrol Dial Transplant. 2004;19:S11-5.

25. Kwon OJ, Lee HG, Kwak JY. The impact of donor and recipient age on the outcome of kidney transplantation. Transplant Proc. 2004;36:20435.

26. Marconi L, Figueiredo A, Campos L, Nunes P, Roseiro A, Parada B, et al. Renal transplantation with donors older than 70 years: Does age matter? Transplant Proc. 2013;45:1251-4.

27. Machado S, Figueiredo N, Neves M, Macário F, Alves R, Mota A, et al. Kidney transplantation using donors over 70 years old: are the criteria for organ allocation too expanded? Transplant Proc. 2012;44:2289-92.

28. Rao PS, Schaubel DE, Wei G, Fenton SS. Evaluating the survival benefit of kidney retransplantation. Transplantation. 2006;82:669-74.

29. Arnol M, Prather JC, Mittalhenkle A, Barry JM, Norman DJ. Long-term kidney regraft survival from deceased donors: risk factors and outcomes in a single center. Transplantation. 2008;86:1084-9.

30. Portal do Serviço Nacional de Saúde. [homepage na Internet]. Ministério da Saúde. [consultado 2017 mar 17]. Disponível em: https://www.sns. gov.pt/.

31. Kostkova P, Brewer H, de Lusignan S, Fottrell E, Goldacre B, Hart G, et al. Who owns the data? Open data for healthcare. Front Public Health. 2016;4:7.

32. Tratamento da Doença Renal Terminal. Sociedade Portuguesa de Nefrologia. [consultado 2017 ago 20]. Disponível em: http://www. spnefro.pt/tratamento_da_doenca_renal_terminal.

33. Registo do Transplante Renal. Sociedade Portuguesa de transplantação. [consultado 2017 ago 20]. Disponível em: http://www.spt.pt/site/desktop/ webpage-58.php.

34. Lima BA, Mendes $M$, Alves $\mathrm{H}$. Measuring kidney transplantation activity. Port J Nephrol Hypert. 2014;28:171-6.

35. Altman DG. The scandal of poor medical research. BMJ. 1994;308:2834. 